
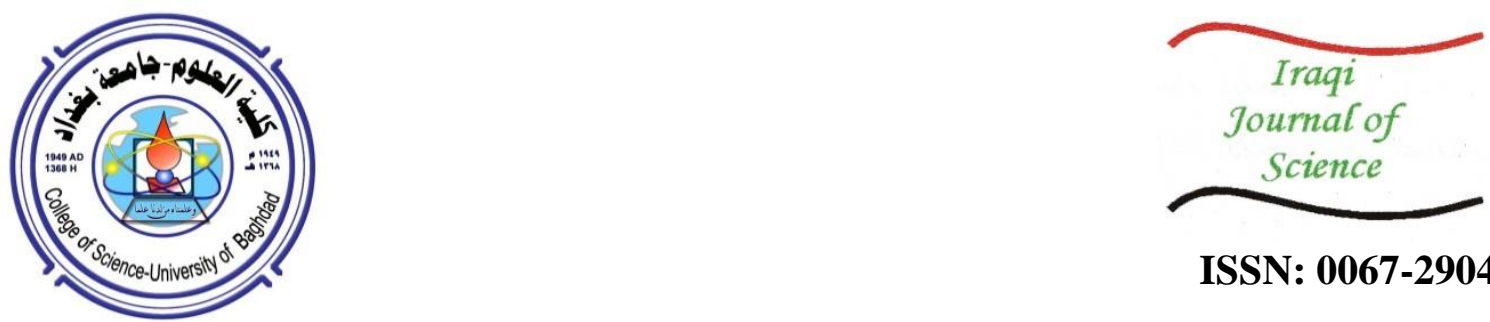

ISSN: 0067-2904

\title{
Subclasses of Analytic Functions of Complex Order Involving Generalized Jackson's (p, q)-derivative
}

\author{
A. Ta. Yousef * Z. Salleh \\ School of informatics and applied mathematics, University Malaysia Terengganu, 21030 Kuala Nerus, \\ Terengganu, Malaysia.
}

Received: 6/10/2019

Accepted: 26/3/2020

\begin{abstract}
This paper aims at introducing a new generalized differential operator and new subclass of analytic functions to obtain some interesting properties like coefficient estimates and fractional derivatives.
\end{abstract}

Keywords: Differential operator, analytic functions, coefficient inequality, fractional integrals, fractional derivatives.

\section{Introduction}

Let $A$ represents the class of functions of the form

$$
f(z)=z+\sum_{m=2}^{\infty} c_{m} z^{m}
$$

which are analytic in the open unit disk $U=\{z \in \mathrm{C}:|z|<1\}$.

We also consider $T$ as a subclass of $A$ consisting of functions of the form

$$
f(z)=z-\sum_{m=2}^{\infty} c_{m} z^{m}, c_{m} \geq 0, z \in U
$$

The theory of $q$-analysis was introduced and studied by Silverman [1]. The theory of $q$ - analysis has a significant role in different areas of mathematics, such as ordinary fractional calculus, $q$-difference, and q-integral equation. It is a fact that the $q$-difference operator plays a critical role in the theory of hypergeometric series (for more information on $q$-calculus, see [2-8]). Some concepts and definitions of $q$-calculus used in this paper are illustrated.

For $p=0$ and $\mathrm{q}=1$, the Jackson`s $(p, q)$-derivative of a function $f \in A$ is given as follows [9]:

$$
D_{p, q} f(z)=\left\{\begin{array}{ccc}
\frac{f(p z)-f(q z)}{(p-q) z} & \text { for } & z \neq 0 \\
f^{\prime}(0) & \text { for } & z=0
\end{array}\right.
$$

From (1.1), we have

$D_{q} f(z)=1+\sum_{m=2}^{\infty}[m]_{p, q} c_{m} z^{m-1}$

where

*Email: abduljabaryousef@gmail.com 
$[m]_{p, q}=\frac{p^{m}-q^{m}}{(p-q) z}$

is called $(p, q)$ - bracket. Obviously, for a function $h(z)=z^{m}$, we obtain $D_{p, q} h(z)=D_{p, q} z^{m}=\frac{p^{m}-q^{m}}{p-q} z^{m-1}=[m]_{p, q} z^{m-1}$.

We note that for $\mathrm{p}=1$, the Jackson $\mathrm{p}, \mathrm{q}$ - derivative will be reduced to the Jackson $q$-derivative [8].

For $f \in A$, we illustrate the Salagean $(p, q)$-differential operator as follows:

$D_{p, q}^{0} f(z)=f(z)$

$D_{p, q}^{1} f(z)=z D_{p, q}^{n} f(z)$

$D_{p, q}^{n} f(z)=z D_{p, q}^{n}\left(D_{p, q}^{n-1} f(z)\right)$

$D_{p, q}^{n} f(z)=z+\sum_{m=2}^{\infty}[m]_{p, q}^{n} c_{m} z^{m}, \quad\left(n \in \mathrm{N}_{0}, z \in U\right)$

Note that if $p=1$ and $\lim _{q} \rightarrow 1^{-}$, we get the Salagean derivative [5]:

$D^{n} f(z)=z+\sum_{m=2}^{\infty} m^{n} c_{m} z^{m}, \quad\left(n \in \mathrm{N}_{0}, z \in U\right)$

Now, in order to define our generalized operator, let

$D^{0} f(\mathrm{z})=\mathrm{D}_{p, q}^{n} f(\mathrm{z})$

$\mathrm{D}_{p, q}^{1, n} f(\mathrm{z})=(1-\beta(\lambda-\alpha)) \mathrm{D}_{p, q}^{n} f(\mathrm{z})+\beta(\lambda-\alpha) z\left(\mathrm{D}_{p, q}^{n} f(\mathrm{z})\right)^{\prime}+\kappa z^{2}\left(\mathrm{D}_{p, q}^{n} f(\mathrm{z})\right)^{\prime \prime}$

$\mathrm{D}_{p, q}^{2, n} f(\mathrm{z})=(1-\beta(\lambda-\alpha)) \mathrm{D}_{p, q}^{1, n} f(\mathrm{z})+\beta(\lambda-\alpha) z\left(\mathrm{D}_{p, q}^{1, n} f(\mathrm{z})\right)^{\prime}+\kappa z^{2}\left(\mathrm{D}_{p, q}^{1, n} f(\mathrm{z})\right)^{\prime \prime}$

In general, we have

$$
\begin{aligned}
D_{\lambda, \beta, \alpha, p, q}^{\tau, n} f(z)= & (1-\beta(\lambda-\alpha)) D_{\lambda, \beta, \alpha, p, q}^{\tau-1, n} f(z)+\beta(\lambda-\alpha) z\left(D_{\lambda, \beta, \alpha, p, q}^{\tau-1, n} f(z)\right)^{\prime} \\
& +\kappa z^{2}\left(D_{\lambda, \beta, \alpha, p, q}^{\tau-1, n} f(z)\right)^{\prime \prime} \\
= & z+\sum_{m=2}^{\infty}[m]_{p, q}^{n}[1+(m-1)(\beta(\lambda-\alpha)+m \kappa)]^{\tau} c_{m} z^{m}
\end{aligned}
$$

where $\alpha \geq 0, \beta \geq 0, z \in U, \lambda>0, \lambda \neq \alpha, \tau \in \mathrm{N}_{0}$.

It is noted that $D_{\lambda, \beta, \alpha, p, q}^{0,0} f(z)=f(z)$ and $D_{\lambda, \beta, \alpha, p, q}^{1,0} f(z)=z f^{\prime}(z)$. Also, it is noted that when $p=1, \beta=1$ and $\alpha=0$, we get the differential operator $D_{\lambda, p, q}^{\tau, n} f(z)$ which was defined and studied by Al-Hawary et al. [10]. When $p=1$ and $\lim _{q \rightarrow 1^{-}}$, we obtain the differential operator

$D_{\lambda, \beta, \alpha, p, q}^{\tau, n} f(z)=z+\sum_{m=2}^{\infty} m^{n}[1+(m-1)(\beta(\lambda-\alpha)+m \kappa)]^{\tau} c_{m} z^{m}\left(\lambda, \beta>0, \quad \alpha \geq 0\right.$ and $\left.\tau \in \mathrm{N}_{0}\right)$

It is notable that when $m=\kappa=0$, the differential operator $D^{\tau}$, defined by Ibrahim and Darus [11], is obtained. Also, when $\beta=1$ and $\alpha=0$, Al-Oboudi operator [12] is obtained and when $\tau=0$, Salagean differential operator [13] is obtained.

A function $f(z)$ that belongs to $A$ is said to be in the class $S_{\lambda, \beta, \alpha, p, q}^{\tau, n}(b, \gamma)$ if it satisfies 


$$
\begin{gathered}
\operatorname{Re}\left\{1+\frac{1}{b}\left(\frac{z\left(\left(D_{\lambda, \beta, \alpha, p, q}^{\tau, n+1} f(z)\right)\right)}{D_{\lambda, \beta, \alpha, p, q}^{\tau, n} f(z)}+1-2 \gamma\right)\right\} \\
>\zeta \mid+\frac{1}{b}\left(\frac{z\left(\left(D_{\lambda, \beta, \alpha, p, q}^{\tau, n+1} f(z)\right)\right)}{D_{\lambda, \beta, \alpha, p, q}^{\tau, n} f(z)}-1\right),(z \in U)
\end{gathered}
$$

where $0<\gamma \leq 1, \zeta \geq 0, \lambda, \beta>0, \alpha \geq 0, n, \tau \in \mathrm{N}_{0}$ and $b \in \mathrm{C}^{*}=\mathrm{C}-\{0\}$.

Further, we shall define the class $S T_{\lambda, \beta, \alpha, p, q}^{\tau, n}(b, \gamma)$ by

$$
S T_{\lambda, \beta, \alpha, p, q}^{\tau, n}(b, \gamma)=S_{\lambda, \beta, \alpha, p, q}^{\tau, n}(b, \gamma) \cap T
$$

In this paper, some properties like coefficient inequalities, distortion, and closure theorems for functions belonging to the class $S T_{\lambda, \beta, \alpha, p, q}^{\tau, n}(b, \gamma)$ are identified and proved.

\section{Coefficient Inequalities}

In this section, the coefficient inequalities for the class $S T_{\lambda, \beta, \alpha, p, q}^{\tau, n}(b, \gamma)$ are attained.

Theorem 2.1. Let $f(z)$ be defined by (1.2) and $f(\mathrm{z}) \in S T_{\lambda, \beta, \alpha, p, q}^{\tau, n}(\mathrm{~b}, \gamma)$

$\sum_{m=2}^{\infty}\left[\left(m[m]_{p, q}+|b|\right)(1-\zeta)+2 \gamma-\zeta+1\right][1+(m-1)(\beta(\lambda-\alpha)+m \kappa)]^{\tau}[m]_{p, q}^{n}\left|c_{m}\right| \leq 2-2 \gamma+|b|(1-\zeta)$

where $0<\gamma \leq 1, \zeta \geq 0$ and $b \in \mathrm{C}^{*}=\mathrm{C}-\{0\}$.

Proof. Suppose that the inequality (2.1) holds. Then, for $z \in U$, we have

$$
\begin{aligned}
& \operatorname{Re}\left\{1+\frac{1}{b}\left(\frac{z\left(\left(D_{\lambda, \beta, \alpha, p, q}^{\tau, n+1} f(z)\right)\right)}{D_{\lambda, \beta, \alpha, p, q}^{\tau, n} f(z)}+1-2 \gamma\right)\right\} \\
& >\zeta\left|1+\frac{1}{b}\left(\frac{z\left(\left(D_{\lambda, \beta, \alpha, p, q}^{\tau, n+1} f(z)\right)\right)}{D_{\lambda, \beta, \alpha, p, q}^{\tau, n} f(z)}-1\right)\right| \\
& \operatorname{Re}\left\{1+\frac{1}{b}\left(\frac{z(2-2 \gamma)-\sum_{m=2}^{\infty}\left(m[m]_{p, q}+2 \gamma+1\right)[m]_{p, q}^{n}[1+(m-1)(\beta(\lambda-\alpha)+m \kappa)]^{\tau} c_{m} z^{m}}{z-\sum_{m=2}^{\infty}[m]_{p, q}^{n}[1+(m-1)(\beta(\lambda-\alpha)+m \kappa)]^{\tau} c_{m} z^{m}}\right.\right. \\
& +1-2 \gamma)\} \\
& >\zeta\left|1+\frac{1}{b}\left(\frac{\sum_{m=2}^{\infty}\left(m[m]_{p, q}-1\right)[m]_{p, q}^{n}[1+(m-1)(\beta(\lambda-\alpha)+m \kappa)]^{\tau} c_{m} z^{m}}{z-\sum_{m=2}^{\infty}[m]_{p, q}^{n}[1+(m-1)(\beta(\lambda-\alpha)+m \kappa)]^{\tau} c_{m} z^{m}}\right)\right|
\end{aligned}
$$

By letting $z \rightarrow 1^{-}$, we haveThis implies that 


$$
\begin{aligned}
& 1+\frac{1}{b}\left(\frac{(2-2 \gamma)-\sum_{m=2}^{\infty}\left(m[m]_{p, q}+2 \gamma+1\right)[m]_{p, q}^{n}[1+(m-1)(\beta(\lambda-\alpha)+m \kappa)]^{\tau}\left|c_{m}\right|}{1-\sum_{m=2}^{\infty}[m]_{p, q}^{n}[1+(m-1)(\beta(\lambda-\alpha)+m \kappa)]^{\tau}\left|c_{m}\right|}+1-2 \gamma\right) \\
> & \zeta\left[1+\frac{1}{b}\left(\frac{\sum_{m=2}^{\infty}\left(m[m]_{p, q}-1\right)[m]_{p, q}^{n}[1+(m-1)(\beta(\lambda-\alpha)+m \kappa)]^{\tau}\left|c_{m}\right|}{1-\sum_{m=2}^{\infty}[m]_{p, q}^{n}[1+(m-1)(\beta(\lambda-\alpha)+m \kappa)]^{\tau}\left|c_{m}\right|}\right)\right]
\end{aligned}
$$

Hence, some simple computations lead to

$$
\begin{aligned}
& \sum_{m=2}^{\infty}\left[\left(m[m]_{p, q}+|b|\right)(1-\zeta)+2 \gamma-\zeta+1\right][1+(m-1)(\beta(\lambda-\alpha)+m \kappa)]^{\tau}[m]_{p, q}^{n}\left|c_{m}\right| \\
\leq & 2-2 \gamma+|b|(1-\zeta)
\end{aligned}
$$

Conversely, suppose that (2.1) is true for $z \in U$. Then

$$
\begin{aligned}
& \operatorname{Re}\left\{1+\frac{1}{b}\left(\frac{z\left(\left(D_{\lambda, \beta, \alpha, p, q}^{\tau, n+1} f(z)\right)\right)^{\prime}}{D_{\lambda, \beta, \alpha, p, q}^{\tau, n} f(z)}+1-2 \gamma\right)\right\} \\
&>\zeta\left|1+\frac{1}{b}\left(\frac{z\left(\left(D_{\lambda, \beta, \alpha, p, q}^{\tau, n+1} f(z)\right)\right)^{\prime}}{D_{\lambda, \beta, \alpha, p, q}^{\tau, n} f(z)}-1\right)\right|>0
\end{aligned}
$$

if

$$
\begin{aligned}
& 1+\frac{1}{b}\left(\frac{(2-2 \gamma)-\sum_{m=2}^{\infty}\left(m[m]_{p, q}+2 \gamma+1\right)[m]_{p, q}^{n}[1+(m-1)(\beta(\lambda-\alpha)+m \kappa)]^{\tau}\left|c_{m}\right|}{1-\sum_{m=2}^{\infty}[m]_{p, q}^{n}[1+(m-1)(\beta(\lambda-\alpha)+m \kappa)]^{\tau}\left|c_{m}\right|}+1-2 \gamma\right)- \\
& \zeta\left[1+\frac{1}{b}\left(\frac{\sum_{m=2}^{\infty}\left(m[m]_{p, q}-1\right)[m]_{p, q}^{n}[1+(m-1)(\beta(\lambda-\alpha)+m \kappa)]^{\tau}\left|c_{m}\right|}{1-\sum_{m=2}^{\infty}[m]_{p, q}^{n}[1+(m-1)(\beta(\lambda-\alpha)+m \kappa)]^{\tau}\left|c_{m}\right|}\right)\right] \\
& >0
\end{aligned}
$$

That is, if $\sum_{m=2}^{\infty}\left[\left(m[m]_{p, q}+|b|\right)(1-\zeta)+2 \gamma-\zeta+1\right][1+(m-1)(\beta(\lambda-\alpha)+m \kappa)]^{\tau}[m]_{p, q}^{n}\left|c_{m}\right| \leq 2-2 \gamma+|b|(1-\zeta)$ then this gives the required condition.

Corollary 2.2. Let the function $f(z)$, defined by (1.2), be in the class $S T_{\lambda, \beta, \alpha, p, q}^{\tau, n}(b, \gamma)$. Then we have $\left|c_{m}\right| \leq \frac{(2-2 \gamma)+|b|(1-\beta)}{\left[\left(m[m]_{p, q}+|b|\right)(1-\zeta)+2 \gamma-\zeta+1\right][1+(m-1)(\beta(\lambda-\alpha)+m \kappa)]^{\tau}[m]_{p, q}^{n}}$, where $m \geq 2,-1 \leq \gamma<1, \zeta \geq 0$, and $b \in \mathrm{C}^{*}=\mathrm{C}-\{0\}$, with equality for $f(z)=\frac{(2-2 \gamma)+|b|(1-\beta)}{\left[\left(m[m]_{p, q}+|b|\right)(1-\zeta)+2 \gamma-\zeta+1\right][1+(m-1)(\beta(\lambda-\alpha)+m \kappa)]^{\tau}[m]_{p, q}^{n}} z^{m}$. 


\section{Integral Means Inequality}

We need the following lemma to prove some inequalities in integral means for functions belonging to the class $S T_{\lambda, \beta, \alpha, p, q}^{\tau, n}(b, \gamma)$.

Lemma 3.1 [11]. If $f$ and $g$ are analytic in $U$ with $f(z) \prec g(z)$, then for $\tau>0$ and $z=r e^{i \theta}(0<r<1)$,

$$
\int_{0}^{2 \pi}|f(z)|^{\tau} d \theta \leq \int_{0}^{2 \pi}|g(z)|^{\tau} d \theta
$$

\section{Theorem 3.2. Let}

$$
\left\{\left[\left(m[m]_{p, q}+|b|\right)(1-\zeta)+2 \gamma-\zeta+1\right][1+(m-1)(\beta(\lambda-\alpha)+m \kappa)]^{\tau}[m]_{p, q}^{n}\right\}_{m=2}^{\infty}
$$

be a non-decreasing sequence. If $f \in S T_{\lambda, \beta, \alpha, p, q}^{\tau, n}(b, \gamma)$, then

$$
\int_{0}^{2 \pi}\left|f\left(r e^{i \theta}\right)\right|^{\tau} d \theta \leq \int_{0}^{2 \pi}\left|g\left(r e^{i \theta}\right)\right|^{\tau} d \theta \quad(0<r<1, \tau>0)
$$

where

$$
f_{2}(z)=z-\frac{(2-2 \gamma)+|b|(1-\beta)}{\left[\left(2[2]_{p, q}+|b|\right)(1-\zeta)+2 \gamma-\zeta+1\right][1+\beta(\lambda-\alpha)+2 \kappa]^{\tau}[2]_{p, q}^{n}} z^{2}
$$

\section{Proof. Let}

$$
f(z)=z-\sum_{m=2}^{\infty} c_{m} z^{m}=\left(1-\sum_{m=2}^{\infty} c_{m} z^{m-1}\right)
$$

and

$$
f_{2}(z)=z-\frac{(2-2 \gamma)+|b|(1-\beta)}{\left[\left(2[2]_{p, q}+|b|\right)(1-\zeta)+2 \gamma-\zeta+1\right][1+\beta(\lambda-\alpha)+2 \kappa]^{\tau}[2]_{p, q}^{n}} z^{2}
$$

then we prove that

$$
\begin{aligned}
& \int_{0}^{2 \pi}\left|1-\sum_{m=2}^{\infty} c_{m} z^{m-1}\right|^{\tau} d \theta \\
& \leq \int_{0}^{2 \pi}\left|1-\frac{(2-2 \gamma)+|b|(1-\beta)}{\left[\left(2[2]_{p, q}+|b|\right)(1-\zeta)+2 \gamma-\zeta+1\right][1+\beta(\lambda-\alpha)+2 \kappa]^{\tau}[2]_{p, q}^{n}} z\right|^{\tau} d \theta
\end{aligned}
$$

By lemma (3.1), it would be sufficient to show that

$$
1-\sum_{m=2}^{\infty}\left|c_{m}\right| z^{m-1} \prec 1-\frac{(2-2 \gamma)+|b|(1-\beta)}{\left[\left(2[2]_{p, q}+|b|\right)(1-\zeta)+2 \gamma-\zeta+1\right][1+\beta(\lambda-\alpha)+2 \kappa]^{\tau}[2]_{p, q}^{n}} z
$$

If the subordination (3.2) holds true, then there exists an analytic function $\omega$ with $\omega(0)=0$ and $|\omega(z)|<1$, such that

$$
1-\sum_{m=2}^{\infty}\left|c_{m}\right| z^{m-1}=1-\frac{(2-2 \gamma)+|b|(1-\beta)}{\left[\left(2[2]_{p, q}+|b|\right)(1-\zeta)+2 \gamma-\zeta+1\right][1+\beta(\lambda-\alpha)+2 \kappa]^{\kappa}[2]_{p, q}^{n}} \omega(z)
$$

Using (2.1), we have 


$$
\begin{aligned}
|\omega(z)| & =\left|\sum_{m=2}^{\infty} \frac{\left[\left(2[2]_{p, q}+|b|\right)(1-\zeta)+2 \gamma-\zeta+1\right][1+\beta(\lambda-\alpha)+2 \kappa]^{\tau}[2]_{p, q}^{n}}{(2-2 \gamma)+|b|(1-\beta)} c_{m} z^{m-1}\right| \\
& \leq|z| \sum_{m=2}^{\infty} \frac{\left[\left(2[2]_{p, q}+|b|\right)(1-\zeta)+2 \gamma-\zeta+1\right][1+\beta(\lambda-\alpha)+2 \kappa]^{\tau}[2]_{p, q}^{n}}{(2-2 \gamma)+|b|(1-\beta)}\left|c_{m}\right| \\
& \leq|z|<1
\end{aligned}
$$

which proves the subordination (3.2).

\section{Fractional Calculus}

In the literature employed by this study, many significant definitions of fractional calculus were illustrated [14-18]. Some important definitions that were used before by Owa [19] and Srivastava [20] are recalled.

Definition 4.1. The fractional integral of order $\mu$ is defined, for a function $f(z)$, by

$D_{z}^{-\mu} f(z)=\frac{1}{\Gamma(\mu)} \int_{0}^{z} \frac{f(\theta)}{(z-\theta)^{1-\mu}} d \theta$

where $\mu>0, f(z)$ is an analytic function in a simply-connected region of the $z$ - plane containing the origin, and the multiplicity of $(z-\theta)^{1-\mu}$ is removed by requiring $\log (z-\theta)$ to be real when $z-\theta>0$.

Definition 4.2. The fractional derivative of order $\mu$ is defined, for a function $f(z)$, by

$D_{z}^{\mu} f(z)=\frac{1}{\Gamma(1-\mu)} \frac{d}{d z} \int_{0}^{z} \frac{f(\theta)}{(z-\theta)^{\mu}} d \theta$

where $0 \leq \mu<1, f(z)$ is an analytic function in a simply-connected region of the $z \&$ plane containing the origin, and the multiplicity of $(z-\theta)^{-\mu}$ is removed as in Definition (4.1) above.

Definition 4.3. Under the hypotheses of definitions (4.2), the fractional derivative of order $m+\mu$ is defined by

$D_{z}^{m+\mu} f(z)=\frac{d}{d \theta} D_{z}^{\mu} f(z)$

where $0 \leq \mu<1$, and $m \in \mathrm{N}_{0}$.

Theorem 4.4. Let $f(z) \in S T_{\lambda, \beta, \alpha, p, q}^{\tau, n}(b, \gamma)$, then

$\left|D_{z}^{-\mu} f(z)\right| \geq \frac{|z|^{1+\mu}}{\Gamma(2+\mu)}$

$$
\left\{1-\frac{(2-2 \gamma)+|b|(1-\beta)}{(2+\mu)\left[\left(2[2]_{p, q}+|b|\right)(1-\zeta)+2 \gamma-\zeta+1\right][1+(\beta(\lambda-\alpha)+2 \kappa)]^{\tau}[2]_{p, q}^{n}}\right\}
$$

and

$$
\begin{aligned}
\left|D_{z}^{-\mu} f(z)\right| \leq & \frac{|z|^{1+\mu}}{\Gamma(2+\mu)} \\
& \left\{1+\frac{(2-2 \gamma)+|b|(1-\beta)}{(2+\mu)\left[\left(2[2]_{p, q}+|b|\right)(1-\zeta)+2 \gamma-\zeta+1\right][1+(\beta(\lambda-\alpha)+2 \kappa)]^{\tau}[2]_{p, q}^{n}}\right\}
\end{aligned}
$$


(4.2)

for $\mu>0$ and $z \in U$. The results (4.1) and (4.2) are sharp.

Proof. We begin with defining the function $G(z)$ by [10]

$$
\begin{aligned}
G(z) & =\Gamma(2+\mu) z^{-\mu} D_{z}^{-\mu} f(z) \\
& =z-\sum_{m=2}^{\infty} \frac{\Gamma(n+1) \Gamma(2+\mu)}{\Gamma(n+1+\mu)} c_{m} z^{m} \\
& =z-\sum_{m=2}^{\infty} \Omega(m) c_{m} z^{m}
\end{aligned}
$$

where

$\Omega(m)=\frac{\Gamma(m+1) \Gamma(2+\mu)}{\Gamma(m+1+\mu)},(m \geq 2)$

If $\varepsilon_{2} \geq \varepsilon_{1}$, implying that $p+\varepsilon_{2}(n-p) \geq p+\varepsilon_{1}(n-p)$

$0<\Omega(m) \leq \Omega(2)=\frac{\Gamma(3) \Gamma(2+\mu)}{\Gamma(3+\mu)}=\frac{2}{2+\mu}$

Furthermore, it follows from theorem (2.1) that

$$
\sum_{m=2}^{\infty}\left|c_{m}\right| \leq \frac{(2-2 \gamma)+|b|(1-\beta)}{\left[\left(2[2]_{p, q}+|b|\right)(1-\zeta)+2 \gamma-\zeta+1\right][1+(\beta(\lambda-\alpha)+2 \kappa)]^{\tau}[2]_{p, q}^{n}}
$$

Then, by using (4.3) and (4.5), we can see that

$$
\begin{aligned}
|G(z)| & \geq|z|-\Omega(2)|z|^{2} \sum_{m=2}^{\infty}\left|c_{m}\right| \\
& \geq|z|-\frac{2[(2-2 \gamma)+|b|(1-\beta)]}{(2+\mu)\left[\left(2[2]_{p, q}+|b|\right)(1-\zeta)+2 \gamma-\zeta+1\right][1+(\beta(\lambda-\alpha)+2 \kappa)]^{\tau}[2]_{p, q}^{n}}|z|^{2}
\end{aligned}
$$

and

$$
\begin{aligned}
& |G(z)| \leq|z|+\Omega(2)|z|^{2} \sum_{m=2}^{\infty}\left|c_{m}\right| \\
\leq & |z|+\frac{2[(2-2 \gamma)+|b|(1-\beta)]}{(2+\mu)\left[\left(2[2]_{p, q}+|b|\right)(1-\zeta)+2 \gamma-\zeta+1\right][1+\beta(\lambda-\alpha)+2 \kappa]^{\tau}[2]_{p, q}^{n}}|z|^{2}
\end{aligned}
$$

which completes the proof.

Finally, we can easily see that the results (4.1) and (4.2) are sharp for the function $f(z)$ given by

$$
\begin{aligned}
& D_{z}^{-\mu} f(z) \leq \frac{z^{1+\mu}}{\Gamma(2+\mu)} \\
& \left\{1+\frac{(2-2 \gamma)+|b|(1-\beta)}{(2+\mu)\left[\left(2[2]_{p, q}+|b|\right)(1-\zeta)+2 \gamma-\zeta+1\right][1+(\beta(\lambda-\alpha)+2 \kappa)]^{\tau}[2]_{p, q}^{n}}\right\}
\end{aligned}
$$

or

$$
f(z)=z-\frac{(2-2 \gamma)+|b|(1-\beta)}{(2+\mu)\left[\left(2[2]_{p, q}+|b|\right)(1-\zeta)+2 \gamma-\zeta+1\right][1+(\beta(\lambda-\alpha)+2 \kappa)]^{\tau}[2]_{p, q}^{n}} z^{2}
$$


Theorem 4.5. If $f(z) \in S T_{\lambda, \beta, \alpha, p, q}^{\tau, n}(b, \gamma)$, then

$$
\begin{aligned}
\left|D_{z}^{\mu} f(z)\right| \geq & \frac{|z|^{1-\mu}}{\Gamma(2-\mu)} \\
& \left\{1-\frac{(2-2 \gamma)+|b|(1-\beta)}{(2-\mu)\left[\left(2[2]_{p, q}+|b|\right)(1-\zeta)+2 \gamma-\zeta+1\right][1+(\beta(\lambda-\alpha)+2 \kappa)]^{\tau}[2]_{p, q}^{n}}\right\}
\end{aligned}
$$

and

$$
\begin{aligned}
\left|D_{z}^{\mu} f(z)\right| \leq & \frac{|z|^{1-\mu}}{\Gamma(2-\mu)} \\
& \left\{1+\frac{(2-2 \gamma)+|b|(1-\beta)}{(2-\mu)\left[\left(2[2]_{p, q}+|b|\right)(1-\zeta)+2 \gamma-\zeta+1\right][1+(\beta(\lambda-\alpha)+2 \kappa)]^{\tau}[2]_{p, q}^{n}}\right\}
\end{aligned}
$$

for $0 \leq \mu<1$ and $z \in U$. The results (4.7) and (4.8) are sharp.

Proof. Define the function [10] $H(z)$ by

$$
\begin{aligned}
H(z) & =\Gamma(2-\mu) z^{\mu} D_{z}^{\mu} f(z) \\
& =z-\sum_{m=2}^{\infty} \frac{\Gamma(m) \Gamma(2-\mu)}{\Gamma(m+1-\mu)} c_{m} z^{m} \\
& =z-\sum_{m=2}^{\infty} \Psi(m) c_{m} z^{m},
\end{aligned}
$$

where

$$
\Psi(m)=\frac{\Gamma(m) \Gamma(2-\mu)}{\Gamma(m+1-\mu)},(m \geq 2)
$$

It is easy to see that

$$
0<\Psi(m) \leq \Psi(2)=\frac{\Gamma(2) \Gamma(2-\mu)}{\Gamma(3-\mu)}=\frac{1}{2-\mu}
$$

Then, by using (4.5) and (4.9), we have

$$
\begin{aligned}
|H(z)| & \geq|z|-\Psi(2)|z|^{2} \sum_{m=2}^{\infty} m\left|c_{m}\right| \\
& \geq|z|-\frac{(2-2 \gamma)+|b|(1-\beta)}{(2-\mu)\left[\left(2[2]_{p, q}+|b|\right)(1-\zeta)+2 \gamma-\zeta+1\right][1+(\beta(\lambda-\alpha)+2 \kappa)]^{\tau}[2]_{p, q}^{n}}|z|^{2}
\end{aligned}
$$

and

$$
\begin{aligned}
|H(z)| & \leq|z|+\Psi(2)|z|^{2} \sum_{m=2}^{\infty} m\left|c_{m}\right| \\
& \leq|z|+\frac{(2-2 \gamma)+|b|(1-\beta)}{(2-\mu)\left[\left(2[2]_{p, q}+|b|\right)(1-\zeta)+2 \gamma-\zeta+1\right][1+(\beta(\lambda-\alpha)+2 \kappa)]^{\tau}[2]_{p, q}^{n}}|z|^{2}
\end{aligned}
$$

Now, (4.7) and (4.8) follow from (4.9) and (4.10), respectively.

Finally, by taking the function $f(z)$ defined by 


$$
\begin{aligned}
\left|D_{z}^{\mu} f(z)\right| \leq & \frac{|z|^{1-\mu}}{\Gamma(2-\mu)} \\
& \left\{1-\frac{(2-2 \gamma)+|b|(1-\beta)}{(2-\mu)\left[\left(2[2]_{p, q}+|b|\right)(1-\zeta)+2 \gamma-\zeta+1\right][1+(\beta(\lambda-\alpha)+2 \kappa)]^{\tau}[2]_{p, q}^{n}}\right\}
\end{aligned}
$$

or for the function given by (4.6), the results (4.7) and (4.8) are easily seen to be sharp.

\section{Fractional Integral Operator}

We need this definition of fractional operator, given by Srivastava et al. [20].

Definition 5.1. For real number $\eta>0, \rho>0$ and $\delta>0$, the fractional integral operator $\mathrm{I}_{0, z}^{\eta, \rho, \delta}$ is defined by

$$
I_{0, z}^{\eta, \rho, \delta} f(z)=\frac{z^{-\eta-\rho}}{\Gamma(\eta)} \int_{0}^{z}(z-t)^{\eta-1} F\left(\eta+\rho,-\delta, \eta, 1-\frac{t}{z}\right) f(t) d t
$$

where a function $f(z)$ is analytic in a simply-connected region of the $z$-plane containing the origin with the order

$$
f(z)=O\left(|z|^{\varepsilon}\right),(z \rightarrow 0)
$$

with $\varepsilon>\max \{0, \gamma-\delta\}-1$.

Here, $F(a, b, c, z)$ is the Gauss hypergeometric function defined by

$$
F(a, b, c, z)=\sum_{m=0}^{\infty} \frac{(a)_{m}(b)_{m}}{(c)_{m}(1)_{m}}
$$

where $(v)_{m}$ is the Pochhammer symbol defined by

$$
(v)_{m}=\frac{\Gamma(v+m)}{\Gamma(v)}=\left\{\begin{array}{cc}
1 & m=0 \\
v(v+1)(v+2) \ldots(v+m-1) & m \in \mathrm{N}
\end{array}\right.
$$

and the multiplicity of $(z-t)^{\eta-1}$ is removed by requiring $\log (z-t)$ to be real when $z-t>0$.

Remark 5.2. For $\rho=-\eta$, we note that

$I_{0, z}^{\eta,-\eta, \delta} f(z)=D_{z}^{-\eta} f(z)$

Lemma 5.3 [21]. If $\eta>0$ and $n>\rho-\delta-1$, then

$$
I_{0, z}^{\eta, \rho, \delta} z^{m}=\frac{\Gamma(m+1) \Gamma(m-\rho+\delta+1)}{\Gamma(m-\rho+1) \Gamma(m+\rho+\delta+1)}
$$

We begin by proving the following.

Theorem 5.4. Let $\eta>0, \rho>2, \eta+\delta>-2, \rho-\delta<2 \quad$ and $\quad \rho(\eta+\delta) \leq 3 \eta$. If $f(z) \in S T_{\lambda, \beta, \alpha, p, q}^{\tau, n}(b, \gamma)$, then

$$
\begin{aligned}
& \left|I_{0, z}^{\eta, \rho, \delta} f(z)\right| \\
\geq & \frac{\Gamma(2-\rho+\delta)|z|^{1-\rho}}{\Gamma(2-\rho) \Gamma(2+\eta+\delta)} \\
& \left\{1-\frac{2(2-\rho+\delta)[(2-2 \gamma)+|b|(1-\beta)]}{(2-\rho)(2+\rho+\delta)\left[\left(2[2]_{p, q}+|b|\right)(1-\zeta)+2 \gamma-\zeta+1\right][1+(\beta(\lambda-\alpha)+2 \kappa)]^{\tau}[2]_{p, q}^{n}}|z|^{2}\right\}
\end{aligned}
$$

and 


$$
\begin{aligned}
& \left|I_{0, z}^{\eta, \rho, \delta} f(z)\right| \\
\leq & \frac{\Gamma(2-\rho+\delta)|z|^{1-\gamma}}{\Gamma(2-\rho) \Gamma(2+\eta+\delta)} \\
& \left\{1+\frac{2(2-\rho+\delta)[(2-2 \gamma)+|b|(1-\beta)]}{(2-\rho)(2+\rho+\delta)\left[\left(2[2]_{p, q}+b \mid\right)(1-\zeta)+2 \gamma-\zeta+1\right][1+(\beta(\lambda-\alpha)+2 \kappa)]^{\tau}[2]_{p, q}^{n}}|z|^{2}\right\}
\end{aligned}
$$

Proof. For $z \in U_{0}$, where

$$
U_{0}=\left\{\begin{array}{cc}
U & , \quad \rho \leq 1 \\
U-\{0\} & , \quad \rho>1
\end{array}\right.
$$

the equalities in (5.1) and (5.2) are attained for the function $f(\mathrm{z})$ given by (4.6).

By using Lemma 5.3, we have

$$
\begin{aligned}
& \quad I_{0, z}^{\eta, \rho, \delta} f(z) \\
& =\frac{\Gamma(2-\rho+\delta)}{\Gamma(2-\rho) \Gamma(2+\eta+\delta)} z^{1-\rho}- \\
& \\
& \qquad \sum_{n=2}^{\infty} \frac{\Gamma(n+1) \Gamma(n-\rho+\delta+1)}{\Gamma(n-\rho+1) \Gamma(n+\eta+\delta+1)} c_{m} z^{m-\rho} \\
& \text { where } z \in U_{0} . \\
& \text { Let } \\
& \Delta(z)=\frac{\Gamma(2-\rho) \Gamma(2+\eta+\delta)}{\Gamma(2-\rho+\delta)} z^{\rho} I_{0, z}^{\eta, \rho, \delta} f(z) \\
& \quad=z-\sum_{m=2}^{\infty} \varphi(m) c_{m} z^{m}
\end{aligned}
$$

where

$$
\varphi(m)=\frac{(2-\rho+\delta)_{m-1}(2)_{m-1}}{(2-\rho)_{m-1}(2+\rho+\delta)_{m-1}},(m \geq 2)
$$

The function $\varphi(m)$ is non-increasing for $n \geq 2$, then we get

$$
\begin{aligned}
|\Delta(z)| \geq & |z|-\varphi(2)|z|^{2} \sum_{m=2}^{\infty}\left|c_{m}\right| \\
& \geq|z|- \\
& \frac{2(2-\rho+\delta)[(2-2 \gamma)+|b|(1-\beta)]}{(2-\rho)(2+\rho+\delta)\left[\left(2[2]_{p, q}+|b|\right)(1-\zeta)+2 \gamma-\zeta+1\right][1+(\beta(\lambda-\alpha)+2 \kappa)]^{\tau}[2]_{p, q}^{n}}|z|^{2}
\end{aligned}
$$

and

$$
\begin{aligned}
|\Delta(z)| \leq & |z|+\varphi(2)|z|^{2} \sum_{m=2}^{\infty}\left|c_{m}\right| \\
& \leq|z|+ \\
& \frac{2(2-\rho+\delta)[(2-2 \gamma)+|b|(1-\beta)]}{(2-\rho)(2+\rho+\delta)\left[\left(2[2]_{p, q}+|b|\right)(1-\zeta)+2 \gamma-\zeta+1\right][1+(\beta(\lambda-\alpha)+2 \kappa)]^{\tau}[2]_{p, q}^{n}}|z|^{2}
\end{aligned}
$$




\section{References}

[1] Silverman, H. "Univalent functions with negative coefficients". Proceedings of the American Mathematical Society, vol. 51, no. 1, pp. 109-116, 1975.

[2] Araci, S., Duran, U., Acikgoz, M., \& Srivastava, H. M. "A certain (p, q) (p, q) -derivative operator and associated divided differences". Journal of Inequalities and Applications, vol. 2016, no. 1, pp. 301, 2016.

[3] Aral, A., Gupta, V., \& Agarwal, R. P. 2013. Applications of q-calculus in operator theory , New York: Springer, 2013, 262p.

[4] Jackson, F. H. "XI. ---on q-functions and a certain difference operator". Earth and Environmental Science Transactions of the Royal Society of Edinburgh, vol. 46, no. 2, pp. 253-281, 1909.

[5] Govindaraj, M., \& Sivasubramanian, S. "On a class of analytic functions related to conic domains involving q-calculus". Analysis Mathematica, vol. 43, no. 3, pp. 475-487, 2017.

[6] Kanas, S., \& Răducanu, D. "Some class of analytic functions related to conic domains". Mathematica slovaca, vol. 64, no. 5, pp. 1183-1196, 2014.

[7] Karahuseyin, Z., Altnkaya, S., \& Yalcn, S. "On H3 (1) Hankel determinant for univalent functions defined by using q- derivative operator", Transylv. J. Math. Mech, vol. 9, no. 1, pp. 2533, 2017.

[8] Srivastava, H. M. "Some generalizations and basic (or q-) extensions of the Bernoulli", Euler and Genocchi polynomials. Appl. Math. Inform. Sci, vol. 5, no. 3, pp. 390-444, 2011.

[9] Saigo, M. "A remark on integral operators involving the Gauss hypergeometric functions". Kyushu Univ., vol.11, pp. 135-143, 1978.

[10] Al-Hawary, T., Yousef, F., \& Frasin, B. A. "Subclasses of Analytic Functions of Complex Order Involving Jackson's (p, q)-derivative”., 2018. Available at SSRN 3289803.

[11] Darus, M., \& Ibrahim, R. W. "On subclasses for generalized operators of complex order". Far East Journal of Mathematical Sciences, vol. 33, no. 3, pp. 299-308, 2009.

[12] Al-Oboudi, F. M. "On univalent functions defined by a generalized Sălăgean operator". International Journal of Mathematics and Mathematical Sciences, vol. 2004, no. 27, pp. 14291436, 2004.

[13] Salagean, G. S. Subclasses of univalent functions, Complex analysis-fifth Romanian-Finnish seminar, Part 1 (Bucharest, 1981), 362-372. Lecture Notes in Math, 1013, 1983.

[14] Aouf, M. K. "On fractional derivatives and fractional integrals of certain subclasses of starlike and convex functions". Math. Japon., vol. 35, no. 5, pp. 831-837, 1990.

[15] Erd' elyi, W. Magnus, F. Oberhettinger and F.G. Tricemi, Tables of integral Transforms, voll. II, McGraw-Hill Book Co., NewYork, Toronto and London,1954.

[16] Oldham, K., \& Spanier, J. The fractional calculus theory and applications of differentiation and integration to arbitrary order (Vol. 111). Elsevier, 1974.

[17] Owa, S., Saigo, M., \& Srivastava, H. M. "Some characterization theorems for starlike and convex functions involving a certain fractional integral operator". Journal of mathematical analysis and applications, vol. 140, no. 2, pp. 419-426, 1989.

[18] Samko, S. G., Kilbas, A. A., \& Marichev, O. I.. Integrals and derivatives of fractional order and some of their applications, 1987.

[19] Owa, S. "On the distribution theorems I". Kyungpook Math. J., vol. 18, pp. 53-59, 1978.

[20] Srivastava, H. M., Saigo, M., \& Owa, S. "A class of distortion theorems involving certain operators of fractional calculus". Journal of mathematical analysis and applications, vol. 131, no. 2, pp. 412-420, 1988.

[21] Srivastava, H. M., \& Owa, S. Univalent functions, fractional calculus, and their applications. Ellis Horwood; New York; Toronto: Halsted Press, 1989. 